\title{
Explicit Upper Bound for Entropy Numbers
}

\author{
Stanislav Hencl
}

\begin{abstract}
We give an explicit upper bound for the entropy numbers of the embedding $I: W^{r, p}\left(Q^{l}\right) \rightarrow C\left(\overline{Q^{l}}\right)$ where $Q^{l}=(-l, l)^{m} \subset \mathbb{R}^{m}, r \in \mathbb{N}, p \in(1, \infty)$ and $r p>m$.
\end{abstract}

Keywords: entropy numbers, embeddings of Sobolev spaces

MSC 2000: Primary 41A46, secondary 46E35

\section{Introduction}

It is a well known fact that the embedding $I: W^{r, p}(\Omega) \rightarrow C(\bar{\Omega})$ is compact if $\Omega$ is a bounded domain in $\mathbb{R}^{m}$ with Lipschitz boundary, $r \in \mathbb{N}, p \in(1, \infty)$ and $r p>m$.

Birman and Solomyak [1] proved that the entropy numbers of this embedding satisfy $e_{k}(I) \sim k^{-\frac{r}{m}}$ if $\Omega$ is a cube (see Section 2 for the definition of entropy numbers). They have used this fact in a study of the spectrum of integral operators and nonsmooth elliptic boundary value problems. For the history and properties of entropy numbers we refer the reader to the books [2] and [4].

Sometimes it is not enough to know that there is $C>0$ such that $e_{k}(I) \leq$ $C k^{-\frac{r}{m}}$; further knowledge of the value of $C$ can be useful. For example, Cucker and Smale [3] ask for an explicit bound on $C$ in the case of the embedding of the fractional Sobolev space $H^{s}(\Omega)$ in $C(\bar{\Omega})$ (recall that $H^{s}(\Omega)=W^{s, 2}(\Omega)$ if $s \in \mathbb{N})$. Our aim is to find an explicit upper bound for the entropy numbers of the embedding $I: W^{r, p}\left((-l, l)^{m}\right) \rightarrow C\left([-l, l]^{m}\right)$ if $l>0, r \in \mathbb{N}, p \in(1, \infty)$ and $r p>m$. In Theorem 6.2 we give explicit values of constants $\alpha=\alpha(r, p, m, l)>0$ and $\beta=\beta(r, p, m, l)>0$ such that

$$
e_{k}\left(I: W^{r, p}\left((-l, l)^{m}\right) \rightarrow C\left([-l, l]^{m}\right)\right) \leq \frac{\alpha}{(k-\beta)^{\frac{r}{m}}}
$$

if $k>\beta$. This gives an answer to Cucker and Smale's question when $s$ is a natural number and $\Omega$ is a cube. Our proof follows the ideas of Birman and

Stanislav Hencl: Department of Mathematics and Statistics, University of Jyväskylä, P.O. Box 35 (MaD), FIN-40014, Jyväskylä, Finland; hencl@maths.jyu.fi The research was supported by the Marie Curie Fellowship HPMT-CT-2000-00090

ISSN 0232-2064 / \$2.50 (c) Heldermann Verlag Berlin 
Solomyak [1]. We do not claim that the upper bound we obtain is sharp in its dependence on $r, m$ and $p$.

\section{Preliminaries}

By $Q^{l}$ we will always denote a cube $(-l, l)^{m}$. Throughout the paper we will use letters $Q$ and $\Delta$ only for cubes and the term cube only for open cubes with sides parallel to the coordinate axes. The closure of a set $A \subset \mathbb{R}^{m}$ is denoted by $\bar{A}$. We say that a set of cubes $\Theta=\left\{Q_{1}, \ldots, Q_{n}\right\}$ forms a partition of a cube $Q$ if $Q_{i}$ are pairwise disjoint and $\bigcup_{i=1}^{n} \overline{Q_{i}}=\bar{Q}$.

Given any Banach space $X$ we use the notation $B_{X}$ for its unit ball (i.e. $\left.B_{X}=\left\{x \in X:\|x\|_{X}<1\right\}\right)$. Suppose that $Y$ is a Banach space and $A \subset Y$ is a bounded domain. The entropy numbers of the set $A$ are defined by

$$
\varepsilon_{i}(A)=\inf \left\{\varepsilon>0: A \subset \bigcup_{j=1}^{i}\left(y_{j}+\varepsilon B_{Y}\right), y_{j} \in Y\right\}, i \in \mathbb{N} .
$$

Let $T: X \rightarrow Y$ be a bounded linear mapping between two Banach spaces. The entropy numbers $\varepsilon_{i}(T)$ of this mapping are defined by $\varepsilon_{i}(T)=\varepsilon_{i}\left(T\left(B_{X}\right)\right)$. We also define the dyadic entropy numbers by $e_{k}(T)=\varepsilon_{2^{k-1}}(T)$ for $k \in \mathbb{N}$ as usual. We write $\lceil r\rceil$ for the upper integer part of a number $r>0$ (i.e. for the smallest $i \in N$ such that $r \leq i)$.

Given any continuous function $f$ from the cube $U=(-1,1)^{m} \subset \mathbb{R}^{m}$ to $\mathbb{R}$, the following substitution formula is true :

$$
\int_{U} f(x) d x=\int_{\partial U} \int_{0}^{1} r^{m-1} f(r s) d r d \mathcal{H}^{m-1}(s) .
$$

By $\# S$ we denote the number of elements of the set $S$ and by $|A|$ we denote the Lebesgue measure of a set $A \subset \mathbb{R}^{m}$. Let $\mathbb{N}_{0}{ }^{m}$ denote the subset of $\mathbb{R}^{m}$ which is formed by the elements with non-negative integer components. An element $\alpha=\left[\alpha_{1}, \ldots, \alpha_{m}\right] \in \mathbb{N}_{0}^{m}$ is called a multi-index and the length of $\alpha$ is $|\alpha|=\alpha_{1}+\ldots+\alpha_{m}$. If $x \in \mathbb{R}^{m}$ and $\alpha \in \mathbb{N}_{0}^{m}$ then

$$
\alpha !=\alpha_{1} ! \cdots \alpha_{m} ! \text { and } x^{\alpha}=x_{1}^{\alpha_{1}} \cdots x_{m}^{\alpha_{m}} .
$$

We shall need that

$$
\#\left\{\alpha \in \mathbb{N}_{0}^{m}:|\alpha|=r\right\}=\left(\begin{array}{c}
r+m-1 \\
m-1
\end{array}\right) .
$$

This follows from the fact that there is a one to one correspondence between such multi-indices and the number of variants to choose $m-1$ balls from the 
row of $r+m-1$ balls. Number $\alpha_{1}$ corresponds to the number of balls from the beginning to the first chosen ball, number $\alpha_{2}$ corresponds to the the number of balls between the first and the second chosen ball, and so on. It follows that for every $r \geq m$ we have

$$
\begin{aligned}
\#\left\{\beta \in \mathbb{N}_{0}{ }^{m}:\right. & \left.\beta_{i} \geq 1,|\beta|=r\right\} \\
& =\#\left\{\alpha \in \mathbb{N}_{0}{ }^{m}:|\alpha|=r-m\right\}=\left(\begin{array}{c}
r-1 \\
m-1
\end{array}\right) .
\end{aligned}
$$

Given $r \in \mathbb{N}$ and $p \in(1, \infty)$ we will denote by $W^{r, p}(\Omega)$ the corresponding Sobolev space. This space is equipped with the norm

$$
\|u\|_{W^{r, p}(\Omega)}^{p}=\sum_{|\alpha| \leq r} \int_{\Omega}\left|D^{\alpha} u\right|^{p} .
$$

Suppose that $r \in \mathbb{N}, p \in(1, \infty), r p>m$ and $\Omega \subset \mathbb{R}^{m}$ is a bounded domain with Lipschitz boundary. It is a well-known fact that for any $u \in W^{r, p}(\Omega)$ there exists $\tilde{u} \in C(\bar{\Omega})$ such that $u=\tilde{u}$ almost everywhere on $\Omega$. In the whole paper we will use only this representative which is defined and continuous on $\bar{\Omega}$. For $p \in(1, \infty)$ we denote its conjugate index by $p^{*}=p /(p-1)$ (i.e. $\left.\frac{1}{p}+\frac{1}{p^{*}}=1\right)$. Given a function $u \in W^{r, p}(\Omega)$ we define $N_{p}(u, r, \Omega)$ by

$$
\left(N_{p}(u, r, \Omega)\right)^{p}=\sum_{|\alpha|=r} \int_{\Omega}\left|D^{\alpha} u\right|^{p} .
$$

We shall need the following bound for the entropy numbers of the unit ball in a finite-dimensional space from [2, page 9].

Lemma 2.1. Suppose that $E$ is a real $k$-dimensional Banach space. Then for all $i \in \mathbb{N}$

$$
i^{-1 / k} \leq \varepsilon_{i}\left(B_{E}\right) \leq 4 i^{-1 / k}
$$

\section{Semi-additive functions}

Let the non-negative function $J(Q)$ of cubes $Q \subset Q^{l}$ be lower semi-additive, i.e. for every cube $Q \subset Q^{l}$ and for any partition of $Q$ into cubes $Q_{j}$ we have

$$
\sum_{j} J\left(Q_{j}\right) \leq J(Q)
$$

By $J$ we will always denote only those non-negative lower semi-additive functions. Let us be given $J$, a partition $\Theta$ of $Q^{l}$ into cubes and $a>0$. We set

$$
G_{a}(J, \Theta)=\max _{\Delta \in \Theta}|\Delta|^{a} J(\Delta) .
$$


Lemma 3.1. Let $s \in \mathbb{N}, a>0, x_{j} \geq 0$ and $y_{j} \geq 0$ for $j \in\{1, \ldots, s\}$. Suppose that $b>0$ is such that

$$
\sum_{j=1}^{s} x_{j} \leq 1, \sum_{j=1}^{s} y_{j} \leq 1 \text { and } x_{j} y_{j}^{a} \geq b
$$

for every $j \in\{1, \ldots, s\}$. Then $b \leq s^{-(a+1)}$.

Proof. From

$$
\sqrt[s]{x_{1} \cdots x_{s}} \leq \frac{x_{1}+\ldots+x_{s}}{s} \leq \frac{1}{s} \text { and } \sqrt[s]{y_{1} \cdots y_{s}} \leq \frac{y_{1}+\ldots+y_{s}}{s} \leq \frac{1}{s}
$$

it follows that

$b^{s} \leq x_{1} \cdots x_{n}\left(y_{1} \cdots y_{s}\right)^{a} \leq s^{-s} s^{-a s}$.

Theorem 3.1. Suppose that $J$ is a non-negative lower semi-additive function such that $J\left(Q^{l}\right) \leq 1$. Then for every $n \in \mathbb{N}$ we can find a partition $\Theta$ of $Q^{l}$ into cubes such that $\# \Theta \leq n$ and

$$
G_{a}(J, \Theta) \leq c_{1} n^{-(a+1)} \text { where } c_{1}=\left(\frac{2^{2 m}}{1-2^{-\frac{m a}{(a+1)}}}\right)^{a+1}\left|Q^{l}\right|^{a} .
$$

Proof. We will construct a sequence of partitions $\Theta_{i}$ of $Q^{l}$ by induction. Set $\Theta_{0}=\left\{Q^{l}\right\}$. In the $(i+1)$-th step we will divide each cube $\Delta \in \Theta_{i}$ satisfying

$$
|\Delta|^{a} J(\Delta) \geq 2^{-m a} G_{a}\left(J, \Theta_{i}\right)
$$

into $2^{m}$ equal cubes. Now, $\Theta_{i+1}$ consists of undivided cubes from $\Theta_{i}$ and of the new cubes which were created by the division.

Let $n_{i}=\# \Theta_{i}, \delta_{i}=G_{a}\left(J, \Theta_{i}\right)$, and by $s_{i}$ we will denote the number of cubes from $\Theta_{i}$ which satisfy (3.2). Plainly $n_{0}=1$ and

$$
n_{i+1} \leq 2^{m} n_{i}
$$

If we divide a cube $\Delta$ into $2^{m}$ equal cubes $\Delta_{j}$, then obviously

$$
\max _{j}\left|\Delta_{j}\right|^{a} J\left(\Delta_{j}\right) \leq 2^{-m a}|\Delta|^{a} J(\Delta) .
$$

Thanks to (3.2) and (3.4) we obtain

$$
\delta_{i+1} \leq 2^{-m a} \delta_{i}
$$

Now let us focus on those $s_{i}$ cubes $\Delta_{j}$ which are divided in the $(i+1)$-th step. Set $x_{j}=J\left(\Delta_{j}\right), y_{j}=\left|\Delta_{j}\right| /\left|Q^{l}\right|$ for $j \in\left\{1, \ldots, s_{i}\right\}$ and $b=2^{-m a} \delta_{i}\left|Q^{l}\right|^{-a}$. Thanks 
to $\sum_{j=1}^{s_{i}} J\left(\Delta_{j}\right) \leq J\left(Q^{l}\right) \leq 1, \sum_{j=1}^{s_{i}}\left|\Delta_{j}\right| \leq\left|Q^{l}\right|$ and (3.2) we obtain from Lemma 3.1 that

$$
\delta_{i} \leq 2^{m a} s_{i}^{-(a+1)}\left|Q^{l}\right|^{a} .
$$

From the construction it follows that

$$
n_{j+1}=n_{j}+s_{j}\left(2^{m}-1\right)
$$

for every $j \in \mathbb{N}_{0}$, and therefore

$$
n_{i} \leq 2^{m} \sum_{j=0}^{i-1} s_{j}
$$

for every $i \in \mathbb{N}$. For a fixed $t \in \mathbb{N}$ we obtain from (3.5) and (3.6) that for every $0 \leq i \leq t$ we have

$$
s_{i} \leq 2^{-(t-i-1) \frac{m a}{a+1}} \delta_{t}^{-\frac{1}{a+1}}\left|Q^{l}\right|^{\frac{a}{a+1}} .
$$

Together with (3.7) this implies

$$
n_{t} \leq 2^{m}\left(\sum_{j=0}^{t-1} 2^{-(t-j-1) \frac{m a}{a+1}}\right) \delta_{t}^{\frac{-1}{a+1}}\left|Q^{l}\right|^{\frac{a}{a+1}} \leq 2^{m} \frac{1}{1-2^{\frac{-m a}{a+1}}} \delta_{t}^{-\frac{1}{a+1}}\left|Q^{l}\right|^{\frac{a}{a+1}} .
$$

Hence (3.1) gives us

$$
\delta_{i} \leq c_{1} 2^{-m(a+1)} n_{i}^{-(a+1)}
$$

for every $i \in \mathbb{N}$. For $i=0$ this inequality is obvious. Given $n \in \mathbb{N}$, we can find $i \in \mathbb{N}$ such that $n_{i} \leq n<n_{i+1} \leq 2^{m} n_{i}$ thanks to (3.3). Thus $\# \Theta_{i} \leq n$ and (3.8) gives us

$G_{a}\left(J, \Theta_{i}\right)=\delta_{i} \leq c_{1} 2^{-m(a+1)} n_{i}^{-(a+1)} \leq c_{1} n^{-(a+1)}$.

\section{Polynomial approximations}

For a cube $\Delta \subset \mathbb{R}^{m}$ we denote by $P_{r}(\Delta)$ the set of all polynomials on $\Delta$ of degree less or equal to $(r-1)$. By $P_{r}(\Delta, M)$ we will denote the set of all polynomials $v \in P_{r}(\Delta)$ such that $\|v\|_{C(\Delta)}<M$.

Given partition $\Theta$ of $Q^{l}$, we denote by $P_{r}(\Theta)$ the set of functions $g$ such that $g \in P_{r}(\Delta)$ for every $\Delta \in \Theta$. That is, on every cube from the partition the function is possibly a different polynomial of degree less or equal to $(r-1)$.

Definition 4.1. For any cube $\Delta \subset \mathbb{R}^{m}$ and for any $u \in W^{r, p}(\Delta)$ we define

$$
\left(P_{\Delta} u\right)(x)=\frac{\chi_{\bar{\Delta}}(x)}{|\Delta|} \sum_{|\alpha| \leq r-1} \int_{\mathbb{R}^{m}} \chi_{\Delta}(y) \frac{(x-y)^{\alpha}}{\alpha !} D^{\alpha} u(y) d y .
$$

for every $x \in \bar{\Delta}$. Plainly $P_{\Delta} u \in P_{r}(\Delta)$. 
Following the ideas from [4, Lemma 6.1, page 289] we compute the constant in the approximation of a function $u \in W^{r, p}(\Delta)$ by $P_{\Delta} u$.

Lemma 4.1. Let $r \in \mathbb{N}, p \in(1, \infty)$ and $r p>m$. For every cube $\Delta \subset \mathbb{R}^{m}$ and for every $u \in W^{r, p}(\Delta)$ we have

$$
\left\|u-P_{\Delta} u\right\|_{C(\bar{\Delta})} \leq c_{2}|\Delta|^{\frac{r}{m}-\frac{1}{p}} N_{p}(u, r, \Delta)
$$

where

$$
c_{2}=\frac{r}{m} \frac{2^{\frac{m}{p^{*}}}}{\left(p^{*}\left(\frac{r}{m}-\frac{1}{p}\right)\right)^{\frac{1}{p^{*}}}}\left(\sum_{|\alpha|=r} \frac{1}{(\alpha !) p^{p^{*}}}\right)^{\frac{1}{p^{*}}}
$$

Proof. Transformation of coordinates gives us that, without loss of generality, we can suppose $\Delta=\left(-\frac{1}{2}, \frac{1}{2}\right)^{n}$ (i.e. $|\Delta|=1$ ). Since $C^{r}(\bar{\Delta})$ is dense in $W^{r, p}(\Delta)$ and

$$
u_{n} \stackrel{W^{r, p}(\Delta)}{\longrightarrow} u \Longrightarrow\left\{\begin{array}{c}
u_{n} \stackrel{C(\bar{\Delta})}{\longrightarrow} u \\
P_{\Delta} u_{n} \stackrel{C(\bar{\Delta})}{\longrightarrow} P_{\Delta} u
\end{array}\right\}
$$

it is sufficient to prove the lemma only for $u \in C^{r}(\bar{\Delta})$. By Taylor's formula we have for every $x \in \bar{\Delta}$ that

$$
\begin{aligned}
& u(x)-\left(P_{\Delta} u\right)(x) \\
& =\frac{\chi_{\bar{\Delta}}(x)}{|\Delta|} \sum_{|\alpha|=r} \frac{r}{\alpha !} \int_{\mathbb{R}^{m}} \chi_{\Delta}(y) \int_{0}^{1}(1-\tau)^{r-1}(x-y)^{\alpha} D^{\alpha} u(y+\tau(x-y)) d \tau d y .
\end{aligned}
$$

We shall write the above expression as $\sum_{|\alpha|=r} \frac{r}{\alpha !} F_{\alpha}(x)$. The substitution $\tilde{\tau}=$ $(1-\tau)$ and $z=(x-y) \tilde{\tau}$ gives us

$$
\left|F_{\alpha}(x)\right| \leq \chi_{\bar{\Delta}}(x) \int_{\mathbb{R}^{m}} \int_{0}^{1} \chi_{\Delta}\left(x-\frac{z}{\tilde{\tau}}\right) \frac{\left|z^{\alpha}\right|}{\tilde{\tau}^{m+1}}\left|D^{\alpha} u(x-z)\right| d \tilde{\tau} d z .
$$

If $x \in \bar{\Delta}$ and $x-\frac{z}{\tilde{\tau}} \in \Delta$, then $x-z=\tilde{\tau}\left(x-\frac{z}{\tilde{\tau}}\right)+(1-\tilde{\tau}) x \in \Delta$. From $\left|x_{i}\right| \leq \frac{1}{2}$ and $\left|x_{i}-\frac{z_{i}}{\tilde{\tau}}\right|<\frac{1}{2}$ it follows that $\left|\frac{z_{i}}{\tilde{\tau}}\right|<1$. Therefore,

$$
\chi_{\bar{\Delta}}(x) \chi_{\Delta}\left(x-\frac{z}{\tilde{\tau}}\right) \leq \chi_{\Delta}(x-z) \chi_{2 \Delta}\left(\frac{z}{\tilde{\tau}}\right),
$$

and hence (4.3) gives us

$$
\left|F_{\alpha}(x)\right| \leq \int_{\mathbb{R}^{m}}\left(\int_{0}^{1} \frac{\left|z^{\alpha}\right|}{\tilde{\tau}^{m+1}} \chi_{2 \Delta}\left(\frac{z}{\tilde{\tau}}\right) d \tilde{\tau}\right) \chi_{\Delta}(x-z)\left|D^{\alpha} u(x-z)\right| d z
$$

We shall write the right hand side as $\left[g_{\alpha} *\left(\chi_{\Delta}\left|D^{\alpha} u\right|\right)\right](x)$. Denote $U=(-1,1)^{m}=$ $2 \Delta$. For any $z \in \mathbb{R}^{m} \backslash\{0\}$ we can find a unique $s=s(z) \in \partial U$ and $\rho=\rho(z)>0$ such that $z=\rho s$. 
Clearly, for $\tilde{\tau}<\rho(z)$ we have $\frac{z}{\tilde{\tau}} \notin U$. This property and $\chi_{U}\left(\frac{z}{\tilde{\tau}}\right) \leq \chi_{U}(z)$ for $\tilde{\tau}<1$ give us

$$
\begin{aligned}
g_{\alpha}(z) & =\int_{\rho}^{1}\left|z^{\alpha}\right| \tilde{\tau}^{-m-1} \chi_{U}\left(\frac{z}{\tilde{\tau}}\right) d \tilde{\tau} \\
& \leq \frac{1}{m}\left(\left(\frac{1}{\rho}\right)^{m}-1\right) \rho^{|\alpha|}\left|s^{\alpha}\right| \chi_{U}(z) \\
& \leq \frac{1}{m} \rho^{r-m}\left|s^{\alpha}\right| \chi_{U}(z)
\end{aligned}
$$

Thanks to (2.1) we obtain

$$
\begin{aligned}
\left\|g_{\alpha}\right\|_{L^{p^{*}\left(\mathbb{R}^{m}\right)}}^{p^{*}} & \leq m^{-p^{*}} \int_{U} \rho^{p^{*}(r-m)}(z)\left|s^{\alpha}(z)\right|^{p^{*}} d z \\
& \leq m^{-p^{*}} \int_{\partial U}\left|s^{\alpha}\right|^{p^{*}} \int_{0}^{1} \rho^{(r-m) p^{*}+m-1} d \rho d \mathcal{H}^{m-1}(s) \\
& =\frac{m^{-p^{*}}}{(r-m) p^{*}+m} \int_{\partial U}\left|s^{\alpha}\right|^{2} d \mathcal{H}^{m-1}(s) \\
& \leq \frac{m^{-p^{*}}}{(r-m) p^{*}+m} \mathcal{H}^{m-1}(\partial U) \\
& =\frac{m^{-p^{*}}}{m p^{*}\left(\frac{r}{m}-1+\frac{1}{p^{*}}\right)} 2 m 2^{m-1} \\
& =\frac{m^{-p^{*}} 2^{m}}{p^{*}\left(\frac{r}{m}-\frac{1}{p}\right)} .
\end{aligned}
$$

From (4.2), (4.4), Hölder's inequality and (4.5) we have

$$
\begin{aligned}
\left|u(x)-P_{\Delta} u(x)\right| & \leq \sum_{|\alpha|=r} \frac{r}{\alpha !}\left|F_{\alpha}(x)\right| \\
& \leq \sum_{|\alpha|=r} \frac{r}{\alpha !}\left\|g_{\alpha}\right\|_{L^{p^{*}\left(\mathbb{R}^{m}\right)}}|| \chi_{\Delta} D^{\alpha} u \|_{L^{p}\left(\mathbb{R}^{m}\right)} \\
& \leq \frac{r}{m} \frac{2^{\frac{m}{p^{*}}}}{\left(p^{*}\left(\frac{r}{m}-\frac{1}{p}\right)\right)^{\frac{1}{p^{*}}}}\left(\sum_{|\alpha|=r} \frac{1}{\alpha !}\left\|D^{\alpha} u\right\|_{L^{p}(\Delta)}\right) \\
& \leq \frac{r}{m} \frac{2^{\frac{m}{p^{*}}}}{\left(p^{*}\left(\frac{r}{m}-\frac{1}{p}\right)\right)^{\frac{1}{p^{*}}}}\left(\sum_{|\alpha|=r} \frac{1}{(\alpha !)^{p^{*}}}\right)^{\frac{1}{p^{*}}} N_{p}(u, r, \Delta) \\
& =c_{2} N_{p}(u, r, \Delta),
\end{aligned}
$$

which proves the assertion. 
Remark 1. It is possible to have a more explicit bound for $c_{2}$ since

$$
\sum_{|\alpha|=r} \frac{1}{(\alpha !)^{p^{*}}} \leq \sum_{|\alpha|=r} \frac{1}{\alpha !}=\frac{m^{r}}{r !} .
$$

The last equality can be proved by induction with respect to $m$. Denote $A(r, m)=\sum_{|\alpha|=r} \frac{1}{\alpha !}$. Plainly $P(r, 1)=\frac{1}{r !}$. For $m>1$ we have

$$
\begin{aligned}
P(r, m) & =\sum_{i=0}^{r} \frac{1}{i !} P(r-i, m-1) \\
& =\sum_{i=0}^{r} \frac{r !}{r !} \frac{1}{i !(r-i) !}(m-1)^{r-i} \\
& =\frac{1}{r !} \sum_{i=0}^{r}\left(\begin{array}{c}
r \\
r-i
\end{array}\right)(m-1)^{r-i} \\
& =\frac{m^{r}}{r !} .
\end{aligned}
$$

On the other hand, this bound is not very sharp, and for given values of $r, m$ and $p$ the sum is better to compute.

Definition 4.2. Given $u \in W^{r, p}\left(Q^{l}\right)$ and a partition $\Theta$ of $Q^{l}$, we define

$$
\left(P_{\Theta} u\right)(x)=\left(P_{\Delta}\right)(x)
$$

for every $\Delta \subset \Theta$ and $x \in \Delta$. Plainly $P_{\Theta} u \in P_{r}(\Theta)$.

Theorem 4.1. Let $r \in \mathbb{N}, p \in(1, \infty)$ and $r p>m$. For every $n \in \mathbb{N}$ and for every $u \in W^{r, p}\left(Q^{l}\right)$ such that $N_{p}\left(u, r, Q^{l}\right) \leq 1$ there is a partition $\Theta$ of $Q^{l}$ such that $\# \Theta \leq n$ and

$$
\left\|u-P_{\Theta} u\right\|_{L^{\infty}(\Delta)} \leq c_{2} c_{1}^{\frac{1}{p}} n^{-\frac{r}{m}} .
$$

Proof. For every partition of $Q^{l}$ into cubes $\Delta_{j}$ we can use Lemma 4.1 to obtain

$$
\left\|u-P_{\Delta_{j}} u\right\|_{C\left(\Delta_{j}\right)} \leq c_{2}\left|\Delta_{j}\right|^{\frac{r}{m}-\frac{1}{p}} N_{p}\left(u, r, \Delta_{j}\right) .
$$

The function

$$
J(\Delta)=\left(N_{p}(u, r, \Delta)\right)^{p}=\sum_{|\alpha|=r} \int_{\Delta}\left|D^{\alpha} u\right|^{p}
$$

is clearly non-negative and lower semi-additive. Hence, we can use Theorem 3.1 for $a=\frac{p r}{m}-1$ to obtain the partition $\Theta$ such that $\# \Theta \leq n$ and

$$
\left\|u-P_{\Delta} u\right\|_{C(\Delta)} \leq c_{2}\left(|\Delta|^{a} N_{p}^{p}(u, r, \Delta)\right)^{\frac{1}{p}} \leq c_{2} c_{1}^{\frac{1}{p}} n^{-\frac{(a+1)}{p}}
$$

for every $\Delta \in \Theta$. 


\section{Auxiliary estimate of covering numbers}

In this section we prove that it is possible to cover the unit ball of $W^{r, p}\left(Q^{l}\right)$ by a finite number of balls in $C\left(\overline{Q^{l}}\right)$.

Lemma 5.1. Let $Q \subset \mathbb{R}^{m}$ be a cube and $P=P_{r}(\bar{Q}, M)$. Let this set be equipped with the supremum norm as in $C(\bar{Q})$. Then, for every $\varepsilon<M$ the following estimate holds for the number of elements in an optimal $\varepsilon-n e t:$

$$
N_{\varepsilon}(P) \leq\left\lceil\frac{4 M}{\varepsilon}\right\rceil^{c_{3}} \text { where } c_{3}=\left(\begin{array}{c}
r-1+m \\
m
\end{array}\right)
$$

Proof. Without loss of generality, we can suppose that $M=1$. On the Banach space $E$ of polynomials on $\bar{Q}$ of degree less or equal to $r-1$ we use a norm $\|\cdot\|_{C(\bar{Q})}$. From (2.2) we obtain that the dimension of this space is equal to

$$
\#\left\{\alpha \in N_{0}{ }^{m}:|\alpha| \leq r-1\right\}=\#\left\{\alpha \in N_{0}{ }^{m+1}:|\alpha|=r-1\right\}=\left(\begin{array}{c}
r-1+m \\
m
\end{array}\right)=c_{3}
$$

Clearly, for $n=\left\lceil\frac{4}{\varepsilon}\right\rceil^{c_{3}}$ we have $4 n^{\frac{-1}{c_{3}}} \leq \varepsilon$ and therefore we can cover the unit ball by $n$ balls of diameter $\varepsilon$ in view of Lemma 2.1. $C\left(\overline{Q^{l}}\right)$.

We will need an estimate of the norm of the embedding $I: W^{r, p}\left(Q^{l}\right) \rightarrow$

Lemma 5.2. Suppose that $r \in \mathbb{N}, p \in(1, \infty)$ and $r p>m$. Then for every $u \in W^{r, p}\left(Q^{l}\right)$ it holds

$$
\|u\|_{C\left(\overline{Q^{l}}\right)} \leq c_{4}\|u\|_{W^{r, p}\left(Q^{l}\right)}
$$

where

$$
c_{4}=c_{2}\left|Q^{l}\right|^{\frac{r}{m}-\frac{1}{p}}+\left(\sum_{|\alpha| \leq r-1} \frac{1}{(\alpha !)^{p^{*}}}\right)^{\frac{1}{p^{*}}} \max \left(\left|Q^{l}\right|^{-\frac{1}{p}},\left|Q^{l}\right|^{\frac{(r-1)}{m}-\frac{1}{p}}\right) .
$$

Proof. By Lemma 4.1 we have

$$
\begin{aligned}
\|u\|_{C\left(\overline{Q^{l}}\right)} & \leq\left\|u-P_{Q^{l}} u\right\|_{C\left(\overline{Q^{l}}\right)}+\left\|P_{Q^{l}} u\right\|_{C\left(\overline{Q^{l}}\right)} \\
& \leq c_{2}\left|Q^{l}\right|^{\frac{r}{m}-\frac{1}{p}}\|u\|_{W^{r, p}\left(Q^{l}\right)}+\left\|P_{Q^{l}} u\right\| \|_{C\left(\overline{Q^{l}}\right)} .
\end{aligned}
$$

From Definition 4.1 and Hölder's inequality we obtain for every $x \in \overline{Q^{l}}$ that 


$$
\begin{aligned}
\left|P_{Q^{l}} u(x)\right| & \leq \frac{1}{\left|Q^{l}\right|} \sum_{|\alpha| \leq r-1}\left|\int_{Q^{l}} \frac{(x-y)^{\alpha}}{\alpha !} D^{\alpha} u(y) d y\right| \\
& \leq \frac{1}{\left|Q^{l}\right|} \sum_{|\alpha| \leq r-1} \frac{(2 l)^{|\alpha|}}{\alpha !}\left|Q^{l}\right|^{\frac{1}{p^{*}}}\left(\int_{Q^{l}}\left|D^{\alpha} u\right|^{p}\right)^{\frac{1}{p}} \\
& \leq \max \left(\left|Q^{l}\right|^{-\frac{1}{p}},\left|Q^{l}\right|^{\frac{(r-1)}{m}-\frac{1}{p}}\right)\left(\sum_{|\alpha| \leq r-1} \frac{1}{(\alpha !)^{p^{*}}}\right)^{\frac{1}{p^{*}}}\|u\|_{W^{r, p}\left(Q^{l}\right)},
\end{aligned}
$$

which shows the assertion.

Remark 2. Using Remark 1 it is possible to have the more explicit bound

$$
\sum_{|\alpha| \leq r-1} \frac{1}{(\alpha !)^{p^{*}}} \leq \sum_{i=0}^{r-1} \sum_{|\alpha|=i} \frac{1}{\alpha !}=\sum_{i=0}^{r-1} \frac{m^{i}}{i !} \leq e^{m} .
$$

Lemma 5.3. Let $r \in \mathbb{N}, p \in(1, \infty)$ and $r p>m$. Put $\tilde{\varepsilon}_{0}=2 c_{2}\left|Q^{l}\right|^{\frac{r}{m}-\frac{1}{p}}$. Then, there are functions $f_{i} \in C\left(\overline{Q^{l}}\right), i \in\left\{1, \ldots, c_{5}\right\}$ such that

$$
B_{W^{r, p}\left(Q^{l}\right)} \subset \bigcup_{i=1}^{c_{5}}\left(f_{i}+\tilde{\varepsilon}_{0} B_{C\left(\overline{Q^{l}}\right)}\right)
$$

where

$$
c_{5}=\left\lceil 8+\frac{4}{c_{2}}\left(\sum_{|\alpha| \leq r-1} \frac{1}{(\alpha !)^{p^{*}}}\right)^{\frac{1}{p^{*}}} \max \left(\left|Q^{l}\right|^{-\frac{r}{m}},\left|Q^{l}\right|^{-\frac{1}{m}}\right)\right\rceil^{\left(\begin{array}{r}
r-1+m \\
m
\end{array}\right)} .
$$

Proof. Suppose that $u \in B_{W^{r, p}\left(Q^{l}\right)}$. From Lemma 4.1 and Lemma 5.2 we have

$$
\left|P_{Q^{l}} u\right| \leq\left|P_{Q^{l}} u-u\right|+|u| \leq c_{2}\left|Q^{l}\right|^{\frac{r}{m}-\frac{1}{p}}+c_{4} .
$$

Hence, we obtain from Lemma 5.1 that the set

$$
\left\{P_{Q^{l}} u:\|u\|_{W^{r, p}\left(Q^{l}\right)} \leq 1\right\}
$$

has an $\frac{\tilde{\varepsilon}_{0}}{2}$-set in $C\left(\overline{Q^{l}}\right)$ of cardinality at most

$$
C=\left\lceil\frac{8 c_{2}\left|Q^{l}\right|^{\frac{r}{m}-\frac{1}{p}}+8 c_{4}}{\tilde{\varepsilon}_{0}}\right\rceil^{c_{3}}
$$

By Lemma 4.1

$$
\left|P_{Q^{l}} u-u\right| \leq c_{2}\left|Q^{l}\right|^{\frac{r}{m}-\frac{1}{p}}=\frac{\tilde{\varepsilon}_{0}}{2},
$$


and therefore this set serves as an $\tilde{\varepsilon}_{0}$-set for $B_{W^{r, p}\left(Q^{l}\right)}$ as well. By (5.3), (5.1) and $\tilde{\varepsilon}_{0}=2 c_{2}\left|Q^{l}\right|^{\frac{r}{m}-\frac{1}{p}}$ we obtain

$$
C=\left\lceil 8+\frac{4}{c_{2}}\left(\sum_{|\alpha| \leq r-1} \frac{1}{(\alpha !)^{p^{*}}}\right)^{\frac{1}{p^{*}}} \max \left(\left|Q^{l}\right|^{-\frac{r}{m}},\left|Q^{l}\right|^{-1 / m}\right)\right\rceil^{\left(\begin{array}{c}
r-1+m \\
m
\end{array}\right)},
$$

which proves the assertion.

\section{Main result}

In this section we give an explicit upper bound for the entropy numbers of the embedding $I: W^{r, p}\left(Q^{l}\right) \rightarrow C\left(\overline{Q^{l}}\right)$.

Theorem 6.1. Let $r \in \mathbb{N}, p \in(1, \infty), r p>m$ and $\varepsilon>0$. There are $N(\varepsilon) \in \mathbb{N}$ and $f_{i} \in L^{\infty}\left(Q^{l}\right), i \in\{1, \ldots, N(\varepsilon)\}$ such that

$$
\begin{aligned}
& B_{W^{r, p}\left(Q^{l}\right)} \subset \bigcup_{i=1}^{N(\varepsilon)}\left(f_{i}+\varepsilon B_{L^{\infty}\left(Q^{l}\right)}\right) \\
& \log _{2} N(\varepsilon) \leq \log _{2} c_{5}+c_{10}^{\frac{m}{r}} \varepsilon^{-\frac{m}{r}}
\end{aligned}
$$

where

$$
c_{10}=2 c_{2} \frac{2^{2 r}}{\left(1-2^{-m\left(1-\frac{m}{p r}\right)}\right)^{\frac{2 r}{m}}}\left|Q^{l}\right|^{\frac{r}{m}-\frac{1}{p}}\left(2+(r+4)\left(\begin{array}{c}
r-1+m \\
m
\end{array}\right)\right)^{\frac{r}{m}} .
$$

Proof. We will use results and notation from the previous sections. Set $a=$ $\frac{p r}{m}-1$ and define $J$ by formula (4.6). For a given $u \in W^{r, p}\left(Q^{l}\right)$ we construct a sequence of partitions $\Theta_{i}$ of $Q^{l}$ as described in the proof of Theorem 3.1. Set

$$
c_{6}=c_{1} 2^{-m(a+1)}=\left(\frac{2^{m}}{1-2^{-\frac{m a}{(a+1)}}}\right)^{a+1}\left|Q^{l}\right|^{a} .
$$

Along with the characteristic numerical sequences $n_{i}=\# \Theta_{i}$ and $\delta_{i}=G_{a}\left(J, \Theta_{i}\right)$ $=\max _{\Delta \in \Theta_{i}}|\Delta|^{a} J(\Delta)$ we also introduce the sequence

$$
\tilde{\delta}_{i}=c_{6} \min _{0 \leq j \leq i}\left(2^{-a m(i-j)} n_{j}^{-(a+1)}\right), i \in \mathbb{N}_{0} .
$$

From the definition of the numbers $\tilde{\delta}_{i}$ it follows that

$$
n_{j}^{a+1} \leq c_{6} \tilde{\delta}_{i}^{-1} 2^{-a m(i-j)} \quad \text { for } \quad 0 \leq j \leq i
$$


In addition, from (3.8), (3.5), (6.3) and (6.4) we have

$$
\delta_{i} \leq \tilde{\delta}_{i} \leq c_{6} n_{i}^{-(a+1)} .
$$

The sequence $\tilde{\delta}_{i}$ is more regular than $\delta_{i}$ since

$$
2^{-(a+1) m} \tilde{\delta}_{i} \leq \tilde{\delta}_{i+1} \leq 2^{-a m} \tilde{\delta}_{i} .
$$

From (6.4) it follows that

$$
\tilde{\delta}_{i+1}=\min \left(2^{-a m} \tilde{\delta}_{i}, c_{6} n_{i+1}^{-(a+1)}\right) .
$$

The right-hand side inequality in (6.7) follows immediately. The left-hand side inequality has to be verified only in the case

$$
\tilde{\delta}_{i+1}=c_{6} n_{i+1}^{-(a+1)} \geq 2^{-(a+1) m} \tilde{\delta}_{i}
$$

which follows easily from (3.3) and (6.6).

Now let $\nu$ be a fixed number such that $0<\nu<c_{6}$. To each semi additive function $J$ we associate the number $k=k(J, a, \nu) \in \mathbb{N}$ determined by the condition

$$
\tilde{\delta}_{k}<\nu \leq \tilde{\delta}_{k-1}
$$

Let $T_{a}(J, \nu)$ denote the segment $\left\{\Theta_{i}\right\}_{i=1}^{k}$. We unite in one class those (and only those) functions $J$ for which the sequences $T_{a}(J, \nu)$ coincide. The number of classes into which we divide the set of all semi-additive functions will be denoted by $N(a, \nu)$.

Lemma 6.1. For $\nu \in\left(0, c_{6}\right)$

$$
\log _{2} N(a, \nu) \leq c_{7} \nu^{-(a+1)^{-1}}
$$

where

$$
c_{7}=\frac{2^{m}}{1-2^{-\frac{m a}{(a+1)}}}\left(2^{m}+\frac{1}{1-2^{-\frac{m a}{(a+1)}}}\right)\left|Q^{l}\right|^{\frac{a}{a+1}} .
$$

Proof. First we estimate the number $N_{1}(a, \nu)$ of more 'extensive' classes which unite in one those functions $J$ for which the actual sequences of partitions $T_{a}(J, \nu)$ do not necessarily coincide but for which the corresponding numerical sequences $\left\{n_{i}\right\}_{i=1}^{k}, k=k(J, a, \nu)$ do coincide. If we apply (3.3), (6.5) with $i=j=k-1$ and (6.8) we obtain

$$
n_{k}^{a+1} \leq 2^{m(a+1)} n_{k-1}^{a+1} \leq 2^{m(a+1)} c_{6} \tilde{\delta}_{k-1}^{-1} \leq 2^{m(a+1)} c_{6} \nu^{-1} .
$$

Let $n^{\star}$ denote the integer part of the number $2^{m}\left(c_{6} \nu^{-1}\right)^{(a+1)^{-1}}$. The equality

$$
n^{\star}+1=1+\sum_{i=1}^{k}\left(n_{i}-n_{i-1}\right)+\left(n^{\star}+1-n_{k}\right)
$$


associates to each sequence $T_{a}(J, \nu)$ a representation of the number $n^{\star}$ as a sum of $k+1$ positive integer terms. By (2.3) the number of such representations is $\left(\begin{array}{c}n^{\star}-1 \\ k\end{array}\right)<2^{n^{\star}-1}$. Thus

$$
\log _{2} N_{1}(a, \nu)<n^{\star} \leq 2^{m}\left(c_{6} \nu^{-1}\right)^{(a+1)^{-1}}
$$

Now let the sequence $\left\{n_{i}\right\}_{i=1}^{k}$ be fixed. We find an estimate of the number of distinct sequences of partitions $\left\{\Theta_{i}\right\}_{i=1}^{k}=T_{a}(J, \nu)$ for which $\# \Theta_{i}=n_{i}$ for every $i \in\{0, \ldots, k\}$. To do this we note that for a given partition $\Theta_{i}$ the partition $\Theta_{i+1}$ is uniquely determined by knowing which $s_{i}$ cubes are further divided upon formation of $\Theta_{i+1}$. The number of possibilities here is $\left(\begin{array}{l}n_{i} \\ s_{i}\end{array}\right)<2^{n_{i}}$. After $k$ steps this gives us a total range of possibilities numbering less than $2^{n_{0}+\ldots+n_{k-1}}$.

From (6.5) for $i=k-1$ and (6.8) we obtain for $j=0, \ldots, k-1$ that

$$
n_{j} \leq\left(c_{6} \nu^{-1}\right)^{(a+1)^{-1}} 2^{-a m(k-1-j) /(a+1)}
$$

which implies the inequality

$$
n_{0}+\ldots+n_{k-1} \leq\left(c_{6} \nu^{-1}\right)^{(a+1)^{-1}}\left[1-2^{-a m /(a+1)}\right]^{-1}=\hat{n} .
$$

Therefore, $N(a, \nu) \leq N_{1}(a, \nu) 2^{\hat{n}},(6.11)$, (6.12) and (6.3) give us

$$
\begin{aligned}
\log _{2} N(a, \nu) & \leq \log _{2} N_{1}(a, \nu)+\hat{n} \\
& \leq c_{6}^{(a+1)^{-1}}\left(2^{m}+\frac{1}{1-2^{-\frac{a m}{a+1}}}\right) \nu^{-(a+1)^{-1}} \\
& =c_{7} \nu^{-(a+1)^{-1}}
\end{aligned}
$$

which finishes the proof of Lemma 6.1.

Continuation of the proof of Theorem 6.1. Recall that $a=\frac{p r}{m}-1$ and that $J$ is defined by (4.6). Since we are interested only in $u \in B_{W^{r, p}\left(Q^{l}\right)}$ we have $N_{p}\left(u, r, Q^{l}\right) \leq 1$, and therefore we can use the inequality (4.7) from the proof of Theorem 4.1. From the left-hand side inequality in (4.7), the definition of $\delta_{i}$ and (6.6) we have

$$
\left\|u-P_{\Theta_{i}} u\right\|_{L^{\infty}\left(Q^{l}\right)} \leq c_{2} \delta_{i}^{\frac{1}{p}} \leq c_{2} \tilde{\delta}_{i}^{\frac{1}{p}}
$$

for every $i \in \mathbb{N}_{0}$.

For a fixed $\varepsilon>0$ we set

$$
\nu=\left(\frac{\varepsilon}{2 c_{2}}\right)^{p} .
$$

First let us suppose that $\nu<c_{6}$ and define $k=k(J, a, \nu)$ by (6.8). We unite in one class those functions $u$ for which the sequences $T_{a}(J, \nu)$ coincide. The number of such classes is estimated by Lemma 6.1 :

$$
\log _{2} N(a, \nu) \leq c_{7}\left(\frac{2 c_{2}}{\varepsilon}\right)^{\frac{m}{r}}
$$


We select one of those classes (denoting it by $\mathrm{K}$ ) and construct an $\varepsilon$-net for it. First we note that the numbers $n_{i}$ and $\tilde{\delta}_{i}, i=0, \ldots, k$ (but not the numbers $\delta_{i}$ ) coincide for all functions in $K$.

We will construct the $\varepsilon$-net in $L^{\infty}\left(Q^{l}\right)$ by induction. We set $\varepsilon_{i}=2 c_{2} \tilde{\delta}_{i}^{\frac{1}{p}}$ for $i=0, \ldots, k$. Suppose that in the $i$-th step we have constructed for the set $K$ an $\varepsilon_{i}$-net of cardinality $N_{i}$ contained in $P_{r}\left(\Theta_{i}\right)$. Let $u \in K$ and let $w \in P_{r}\left(\Theta_{i}\right)$ be the element of the $\varepsilon_{i}$-net for which $\|u-w\|_{L^{\infty}\left(Q^{l}\right)} \leq \varepsilon_{i}$. Then (6.13) gives us $\left\|P_{\Theta_{i+1}} u-w\right\|_{L^{\infty}\left(Q^{l}\right)} \leq\left\|P_{\Theta_{i+1}} u-u\right\|_{L^{\infty}\left(Q^{l}\right)}+\|u-w\|_{L^{\infty}\left(Q^{l}\right)} \leq c_{2} \tilde{\delta}_{i+1}^{\frac{1}{p}}+\varepsilon_{i} \leq 2 \varepsilon_{i}$. Thus, in each cube $\Delta \in \Theta_{i+1}$ the function $P_{\Theta_{i+1}} u-w$ belongs to the set $P_{r}\left(\Delta, 2 \varepsilon_{i}\right)$. By virtue of Lemma 5.1, definition of $\varepsilon_{i}$ and the left-hand side inequality in (6.7) this set has an $\left(\frac{\varepsilon_{i+1}}{2}\right)$-net whose cardinality does not exceed the number

$$
\left\lceil\frac{16 \varepsilon_{i}}{\varepsilon_{i+1}}\right\rceil^{c_{3}} \leq 2^{(r+4) c_{3}}=c_{8}
$$

where $c_{3}=\left(\begin{array}{c}r-1+m \\ m\end{array}\right)$. Therefore, the set $\left\{P_{\Theta_{i+1}} u: u \in K\right\}$ has an $\left(\frac{\varepsilon_{i+1}}{2}\right)$-net which is contained in $P_{r}\left(\Theta_{i+1}\right)$ and whose cardinality satisfies

$$
N_{i+1} \leq c_{8}^{n_{i+1}} N_{i} .
$$

By (6.13) the same net serves as an $\varepsilon_{i+1}$-net for the set $K$. In this way, starting from $\varepsilon_{0}$-net, we construct an $\varepsilon_{k}$-net with cardinality $N_{k}$ for the set $\mathrm{K}$. By the definition of $\varepsilon_{k},(6.8)$ and (6.14) this is also an $\varepsilon$-net. By (3.3) and (6.12) it holds

$$
N_{k} \leq N_{0} c_{8}^{n_{1}+\ldots+n_{k}} \leq N_{0} c_{8}^{2^{m}\left(n_{0}+\ldots+n_{k-1}\right)} \leq N_{0} c_{8}^{2^{m} \hat{n}}
$$

Clearly, (6.12), (6.14) and (6.3) give us $2^{m} \hat{n}=c_{9} \varepsilon^{-\frac{m}{r}}$ where

$$
\begin{aligned}
c_{9} & =2^{m} c_{6}^{(a+1)^{-1}} \frac{1}{1-2^{-\frac{a m}{a+1}}}\left(2 c_{2}\right)^{\frac{p}{a+1}} \\
& =\left(\frac{2^{m}}{1-2^{-\frac{a m}{a+1}}}\right)^{2}\left|Q^{l}\right|^{\frac{a}{a+1}}\left(2 c_{2}\right)^{\frac{m}{r}} .
\end{aligned}
$$

From definition of $\varepsilon_{0},(6.4), n_{0}=1$ and (6.3) we obtain

$$
\varepsilon_{0}=2 c_{2} \tilde{\delta}_{0}^{\frac{1}{p}}=2 c_{2} c_{6}^{\frac{1}{p}} \geq 2 c_{2}\left|Q^{l}\right|^{\frac{r}{m}-\frac{1}{p}} .
$$

Therefore, $N_{0} \leq c_{5}$ in view of Lemma 5.3. Now we combine the estimate

$$
N_{k} \leq c_{5} c_{8}^{c_{9} \varepsilon^{-\frac{m}{r}}}
$$

which we have obtained for each of the classes with the estimate (6.15) for the number of classes to obtain

$$
\log _{2} N(\varepsilon) \leq \log _{2} c_{5}+\left(\log _{2} c_{8}\right) c_{9} \varepsilon^{-\frac{m}{r}}+c_{7}\left(2 c_{2}\right)^{\frac{m}{r}} \varepsilon^{-\frac{m}{r}} .
$$


Let us return to the case $\nu \geq c_{6}$. Then (6.14) and (6.3) give us

$$
\varepsilon \geq 2 c_{2} c_{6}^{\frac{1}{p}} \geq 2 c_{2}\left|Q^{l}\right|^{\frac{r}{m}-\frac{1}{p}} .
$$

Due to Lemma 5.3 we have $N(\varepsilon) \leq c_{5}$, and hence (6.19) is valid also if $\nu \geq c_{6}$.

Now we can use the definition of the constants involved, namely (6.9), (6.16), (6.18), (5.1), $a=\frac{p r}{m}-1$ and (6.2) to obtain

$$
\begin{aligned}
\left(c_{7}\left(2 c_{2}\right)^{\frac{m}{r}}\right. & \left.+\left(\log _{2} c_{8}\right) c_{9}\right)^{\frac{r}{m}} \\
& \leq 2 c_{2}\left|Q^{l}\right|^{\frac{a r}{(a+1) m}}\left(2\left(\frac{2^{m}}{1-2^{-\frac{m a}{(a+1)}}}\right)^{2}+(r+4) c_{3}\left(\frac{2^{m}}{1-2^{-\frac{m a}{(a+1)}}}\right)^{2}\right)^{\frac{r}{m}} \\
& =c_{10},
\end{aligned}
$$

which completes the proof of Theorem 6.1.

Finally we can state our main result. The values of constants $c_{10}, c_{5}$ and $c_{2}$ are given by (6.2), (5.6) and (4.1). Recall that $\lceil x\rceil$ denotes the upper integer part of a number $x>0$. By Remark 1 and Remark 2 it is possible to have bounds not involving sums.

Theorem 6.2. Let $r \in \mathbb{N}, p \in(1, \infty), r p>m$ and $k>1+\log _{2} c_{5}$. Then

$$
e_{k}\left(I: W^{r, p}\left(Q^{l}\right) \rightarrow C\left(\overline{Q^{l}}\right)\right) \leq \frac{2 c_{10}}{\left(k-1-\log _{2} c_{5}\right)^{\frac{r}{m}}} .
$$

Proof. Given $\varepsilon>0$, fix $f_{i} \in L^{\infty}\left(Q^{l}\right), i \in\{1, \ldots, N(\varepsilon)\}$ as in (6.1) and choose

$$
\tilde{f}_{i} \in A_{i}:=\left(f_{i}+\varepsilon B_{L^{\infty}\left(Q^{l}\right)}\right) \cap C\left(\overline{Q^{l}}\right) .
$$

If $A_{i}=\emptyset$, then set $f_{i} \equiv 0$. Since every function from $W^{r, p}\left(Q^{l}\right)$ is in $C\left(\overline{Q^{l}}\right)$ we obtain from (6.1) that

$$
B_{W^{r, p}\left(Q^{l}\right)} \subset \bigcup_{i=1}^{N(\varepsilon)}\left(\tilde{f}_{i}+2 \varepsilon B_{C\left(\overline{Q^{l}}\right)}\right)
$$

Now the definition of dyadic entropy numbers and the relation $k>1+\log _{2} c_{5}$ give us

$$
\begin{aligned}
e_{k}\left(I: W^{r, p}\left(Q^{l}\right) \rightarrow C\left(\overline{Q^{l}}\right)\right) & \leq \inf \left\{\varepsilon>0: N(\varepsilon / 2) \leq 2^{k-1}\right\} \\
& \leq \inf \left\{\varepsilon>0: \log _{2} c_{5}+c_{10^{\frac{m}{r}}} \varepsilon^{-\frac{m}{r}} 2^{\frac{m}{r}} \leq k-1\right\} \\
& =\frac{2 c_{10}}{\left(k-1-\log _{2} c_{5}\right)^{\frac{r}{m}}}
\end{aligned}
$$

and proves the assertion. 
There are several known ways how to extend a function from a domain with Lipschitz boundary, and the explicit norm of the extension is known in some cases (see [5]). The next theorem gives us a bound for the entropy numbers of the embedding $I: W^{r, p}(\Omega) \rightarrow C(\bar{\Omega})$ if we know the norm of the extension operator.

Theorem 6.3. Let $r \in \mathbb{N}, p \in(1, \infty), r p>m, k>1+\log _{2} c_{5}$ and $\Omega \subset$ $Q_{l} \subset \mathbb{R}^{m}$. Suppose that there exists a linear extension operator $E: W^{r, p}(\Omega) \rightarrow$ $W^{r, p}\left(Q_{l}\right)$ of norm $c_{11}$, i.e.

$$
\|E f\|_{W^{r, p}\left(Q^{l}\right)} \leq c_{11}\|f\|_{W^{r, p}(\Omega)}
$$

for every $f \in W^{r, p}(\Omega)$, and moreover $E f=f$ on $\Omega$. Then

$$
e_{k}\left(I: W^{r, p}(\Omega) \rightarrow C(\bar{\Omega})\right) \leq c_{11} \frac{2 c_{10}}{\left(k-1-\log _{2} c_{5}\right)^{\frac{r}{m}}} .
$$

Proof. For every function $f \in B_{W^{r, p}(\Omega)}$ we have $E f \in c_{11} B_{W^{r, p}\left(Q^{l}\right)}$. By multiplying both sides of $(6.20)$ by $c_{11}$ we see that there is an $i \in\{1, \ldots, N(\varepsilon)\}$ such that

$$
\left\|f-c_{11} \tilde{f}_{i}\right\|_{C(\bar{\Omega})} \leq\left\|E f-c_{11} \tilde{f}_{i}\right\|_{C\left(\overline{Q^{l}}\right)}<c_{11} 2 \varepsilon .
$$

Therefore, analogously to the proof of the previous theorem we obtain the desired bound for entropy numbers.

Acknowledgement. The author wishes to express his thanks to Professor David Edmunds for suggesting the problems and for many stimulating conversations.

\section{References}

[1] Birman, M. S. and M. Z.Solomyak: Quantitative analysis in Sobolev imbedding theorems and applications to spectral theory. Amer. Math. Soc. Transl. 114 (1980)(2), 1 - 132 .

[2] Carl, B. and I. Stephani: Entropy, Compactness and the Approximation of Operators. Cambridge: Cambridge University Press 1990.

[3] Cucker, F. and S. Smale: On the mathematical foundations of learning. Bulletin of the American Mathematical Society 39 (2001), 1 - 49.

[4] Edmunds, D. E. and W. D. Evans: Spectral Theory and Differential Operators. New York: Oxford University Press 1987.

[5] Mikhlin, S. G.: Constants in Some Inequalities of Analysis. Chichester: John Wiley \& Sons 1986.

Received 14.01.2004 